\title{
Single Event Effects Testing of a Commercial-Off-The-Shelf Analog-to-Digital Converter in a Camera Application
}

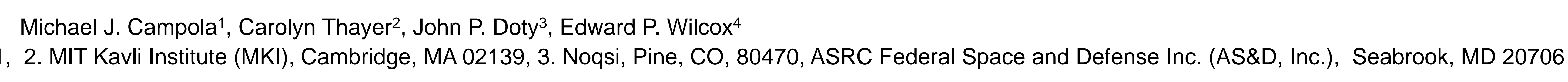

Abstract: Single event effect data is presented on the Analog Devices AD7984. The recent heavy-ion test results showcase application-specific results for the commercial part in its intended application.

\section{Introduction}

Radiation-induced upsets are a concern for Charged particles that deposit energy within the (n)

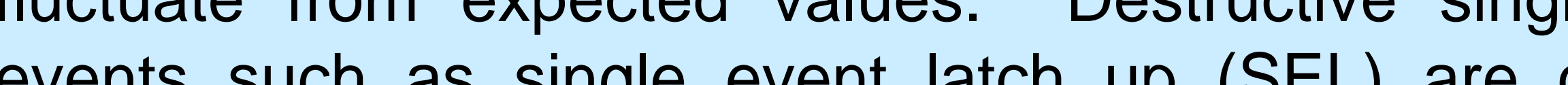
particular concern with commercial off-the-shel (COTS) devices. Many data converters that have been lested have shown varied response to heavy ion

The Analog Devices AD7984 is an 18-bit, (ADC) that operates from a single power supply. It contains a low-power, high-speed, 18-bit sampling $A D C$ and a versatile serial interface port [5].

TESS Mission Overview

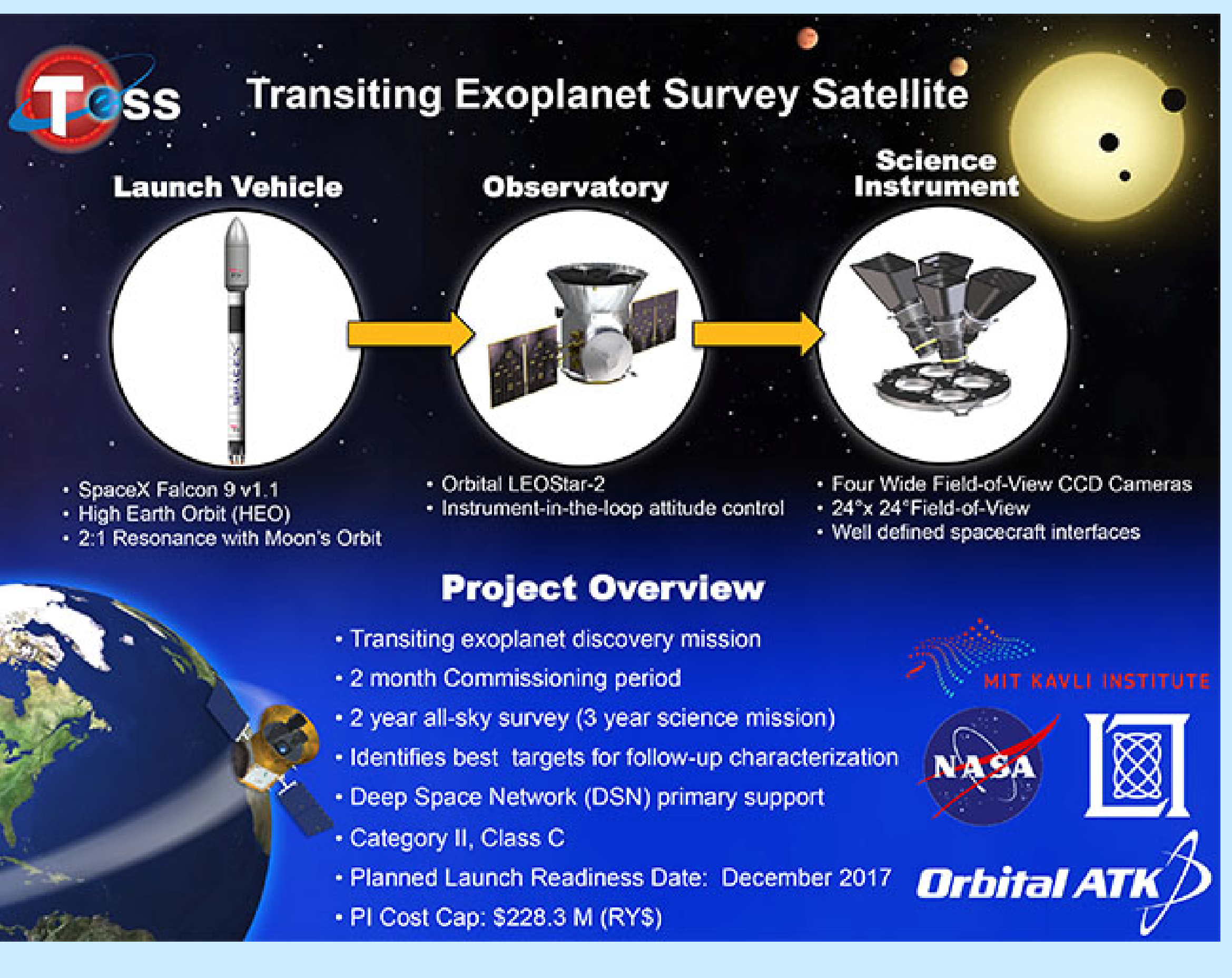

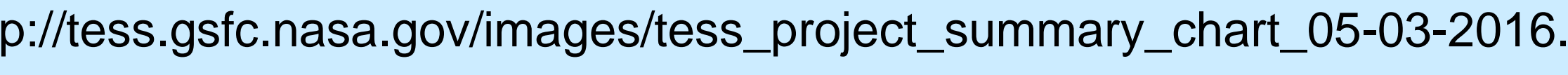

- Highly Eliptical Orbit 15-59 Earth Radii

- Launching During Solar Minimum

4 Cameras, Wide field of View

- Measuring transiting planets of other stars

- 2 Year Mission

- Low TID, focus on SEE
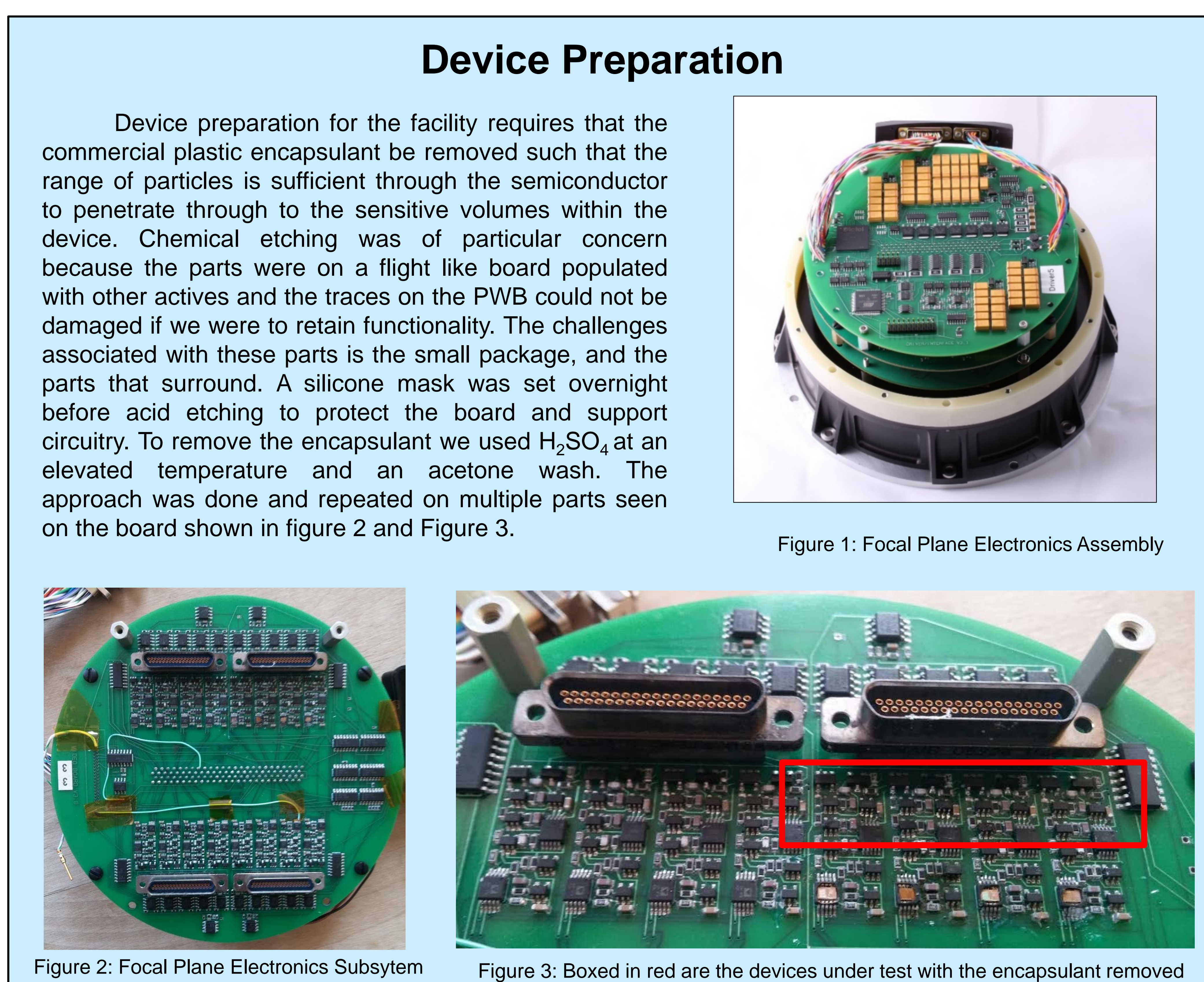

igure 2: Focal Plane Electronics Subsyter
board that houses the ADT984

to are the expices under test with the
to expose the die for heary ion testing

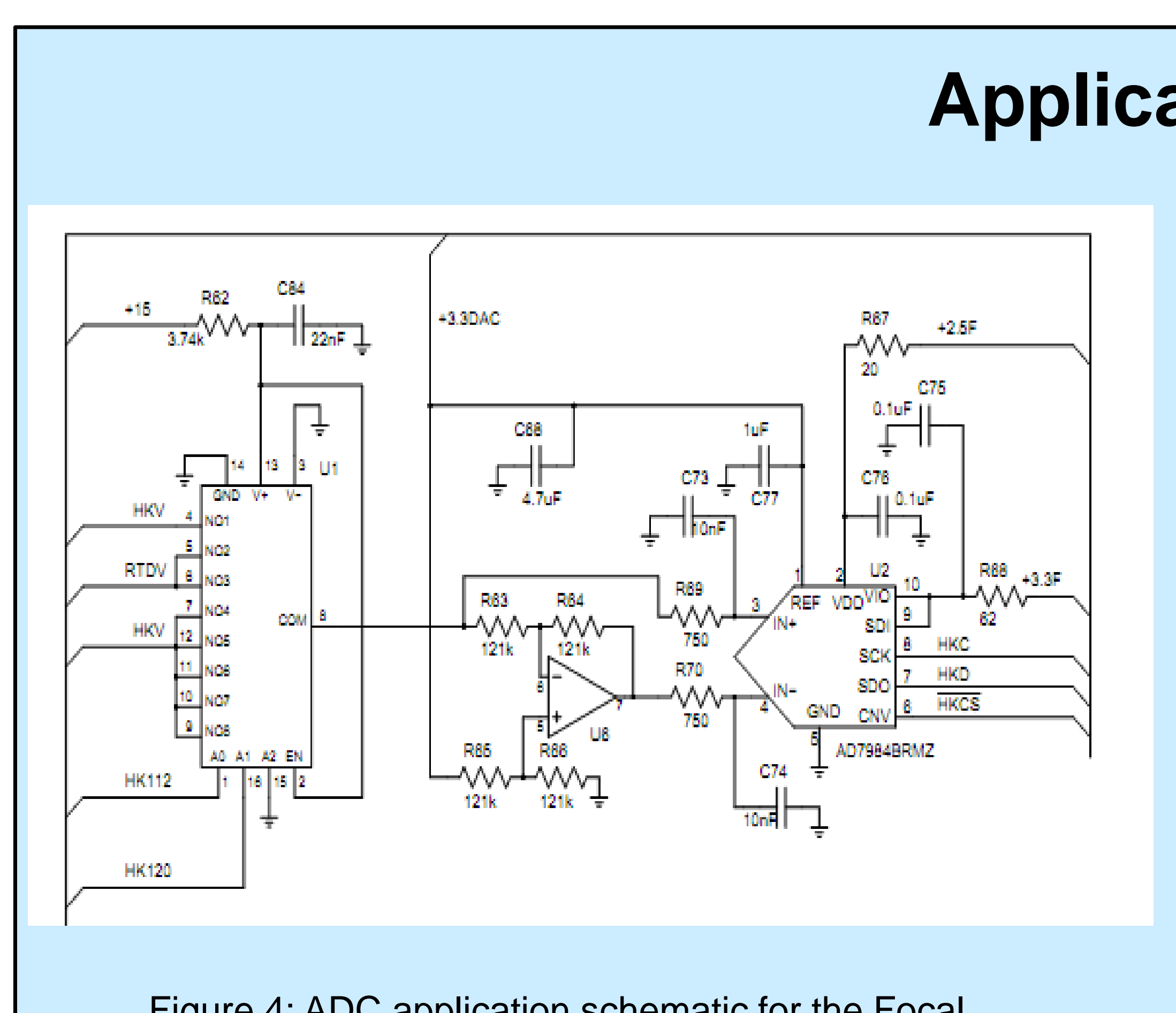

Figure 4: ADC application schematic for the Focal
Plane Electronics Subsytem

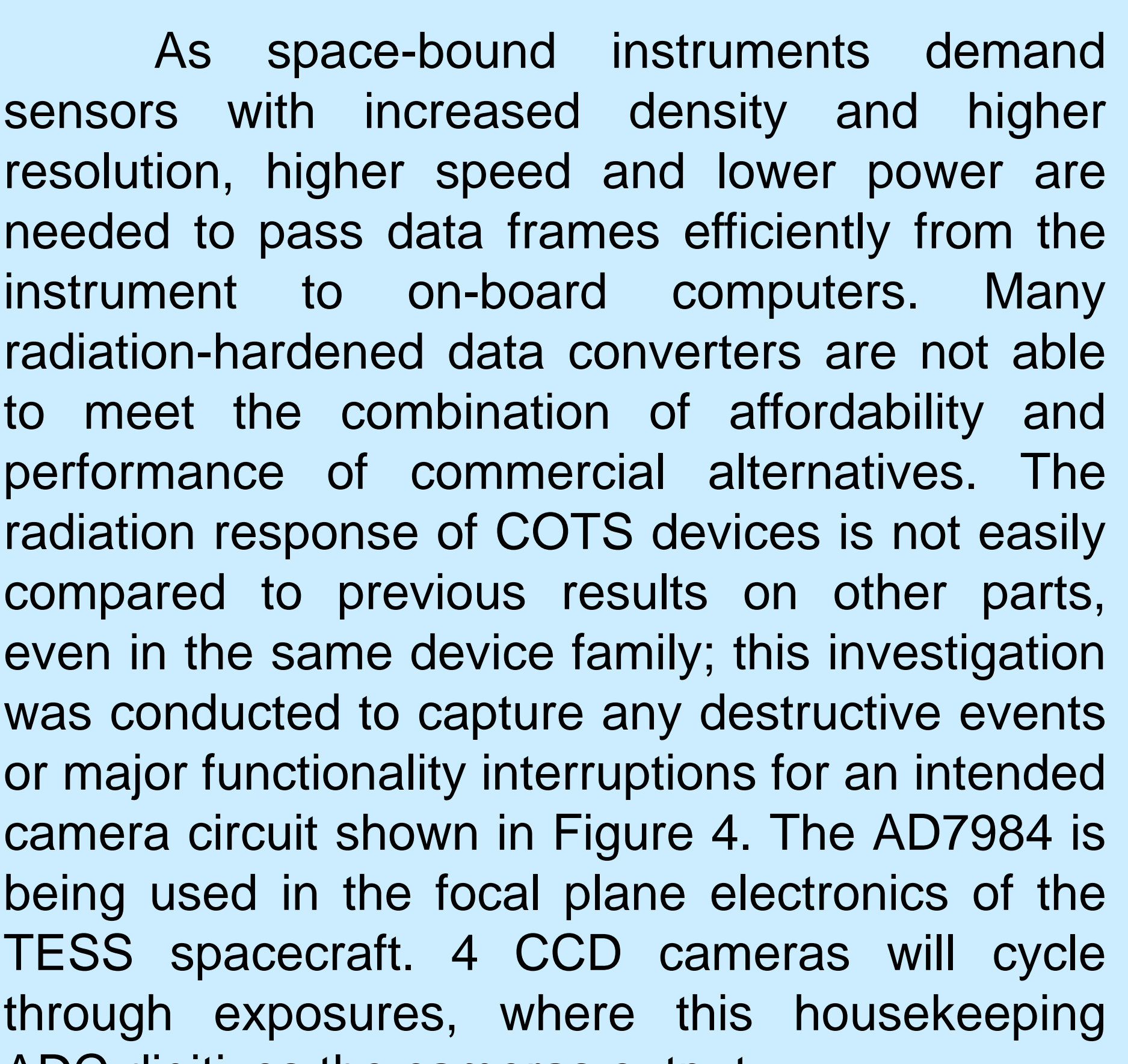

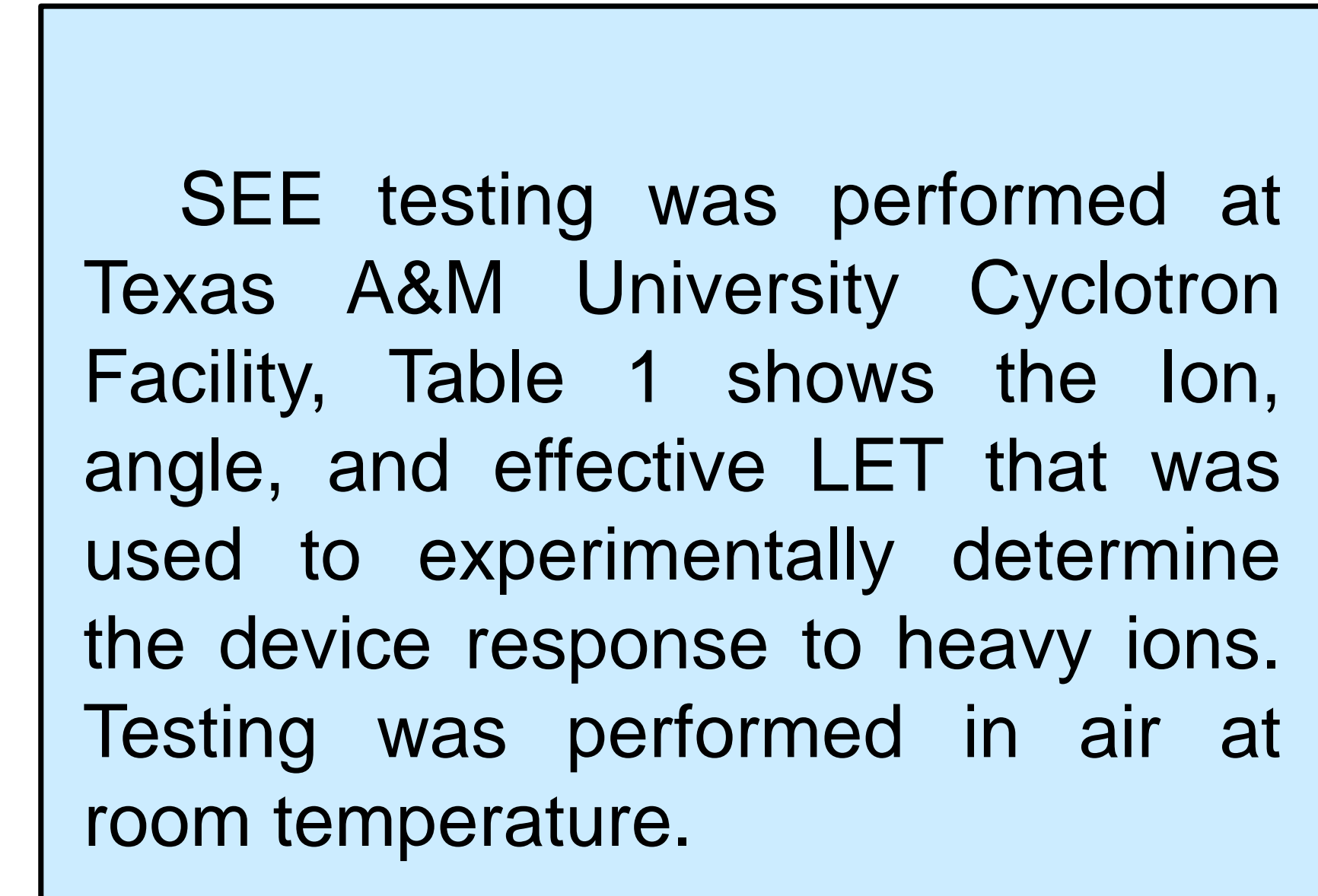

Test Facility

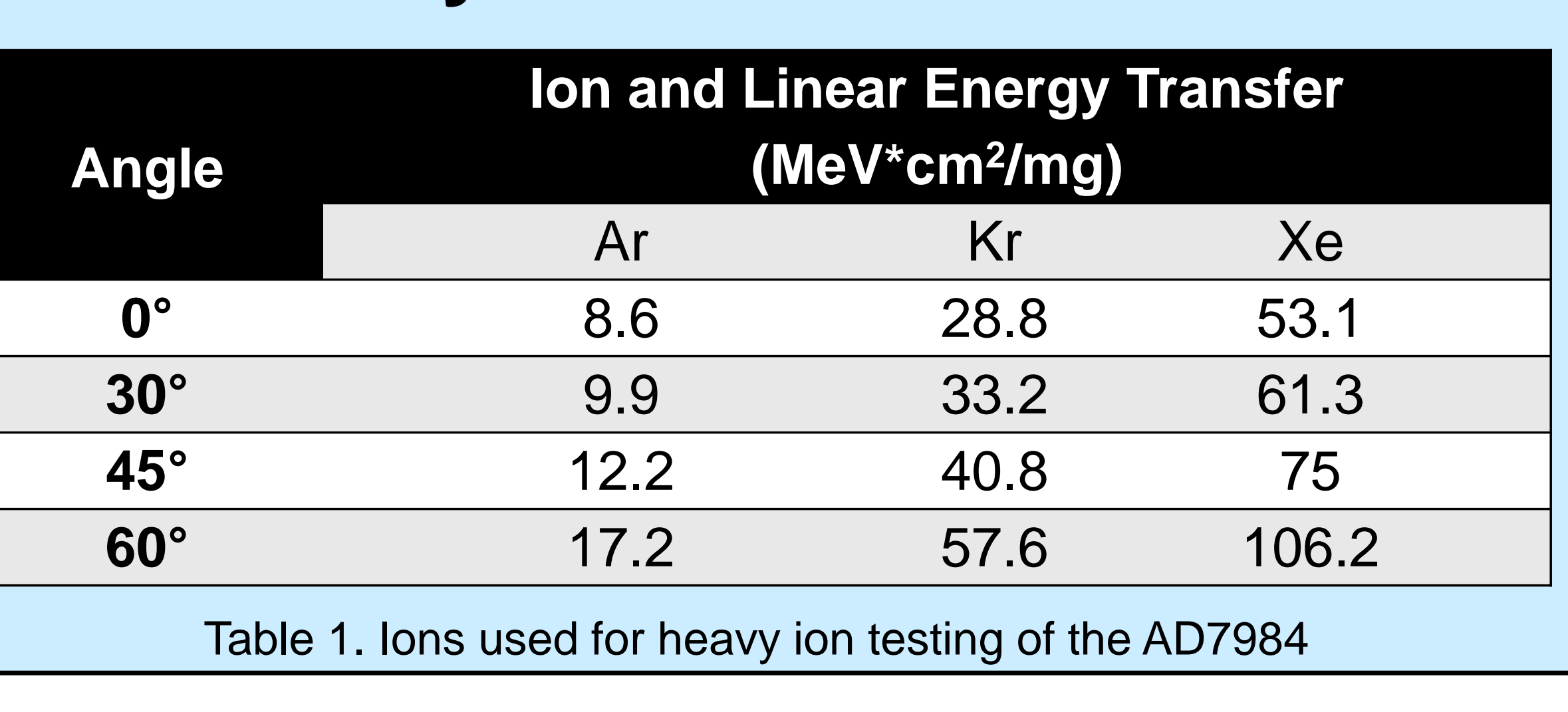

Test Results and Discussion

The parts were tested for a given LET to a predetermined fluence of ions. During beam
exposure the current levels and an image output were monitored from the control room.
After each ion beam exposure post processing of the image file was done to determine the count of single events. For this test, a single containing more than 10 pixels at least 10 standard deviations beyond the noise floor data was then scaled to 100 frames and the results are plotted in Figure 2. The Weibull fit to the data has an onset of $23.4 \mathrm{MeV} . \mathrm{cm}^{2} / \mathrm{mg}$, the last ion LET where no upset was recorded, and
an estimated saturation of $3 \times 10^{-4} \mathrm{~cm}^{2} /$ device. an estimated saturation of $3 \times 10^{-4} \mathrm{~cm}^{2} / \mathrm{dev}$ ice. For the given application there were two types of radiation responses: short output transients (glitches seen at the beginning and end of the rising edge) and large scale transients that correspond with false readings of saturation on the input. No destructive events were recorded. Supply voltage to the system was not varied from nominal applicatio Ievels. Figure 5 shows dignal ouput levels of the ADC following a single event heavy-ion lower bound for the amplitude of the captured Joper

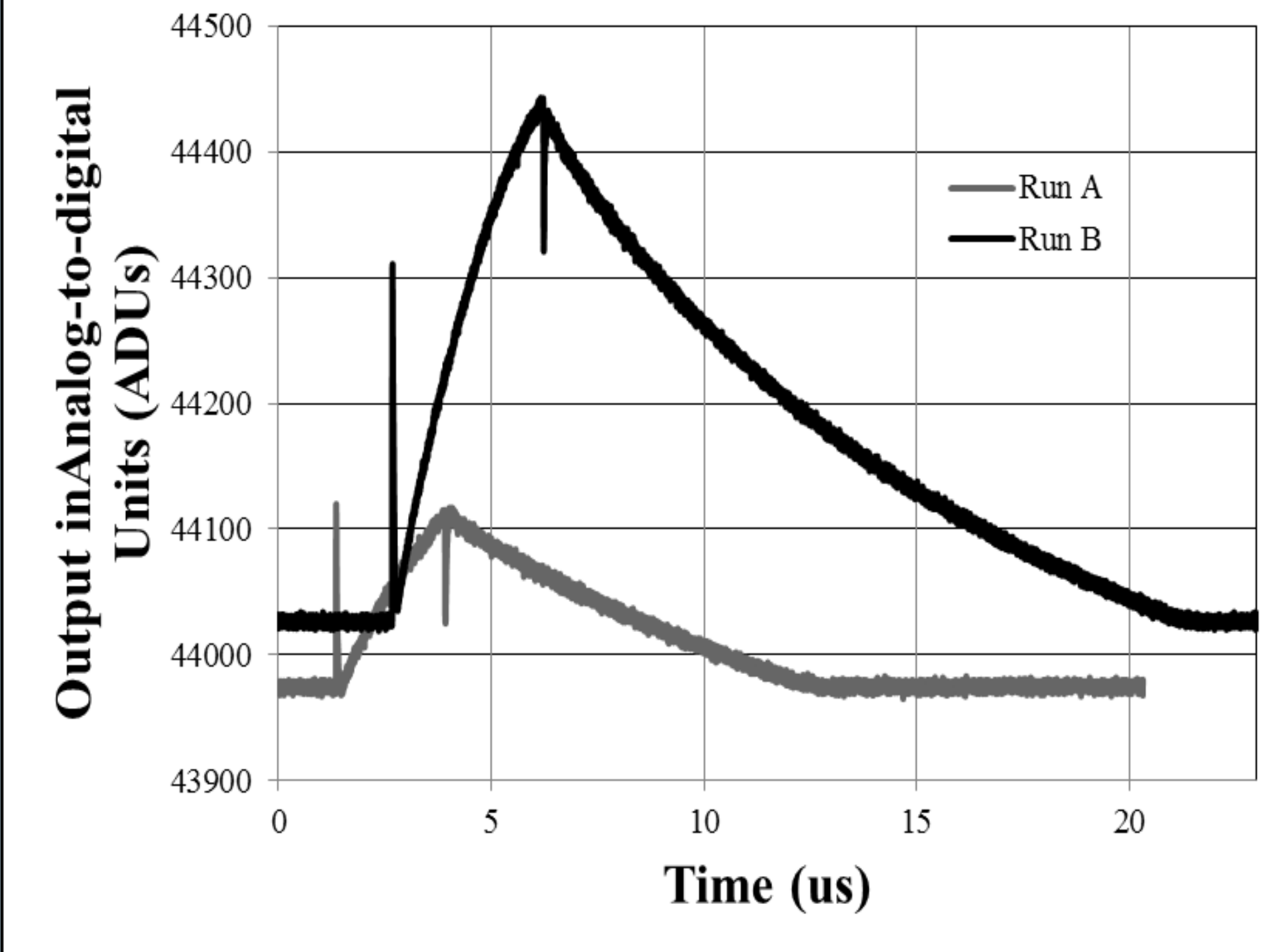

Figure 5: Baard response to ingle event heavy ion strike
tor wo difterent runs

For the single event rate calculations on the had $>10$ errors, a requirement for the mission.

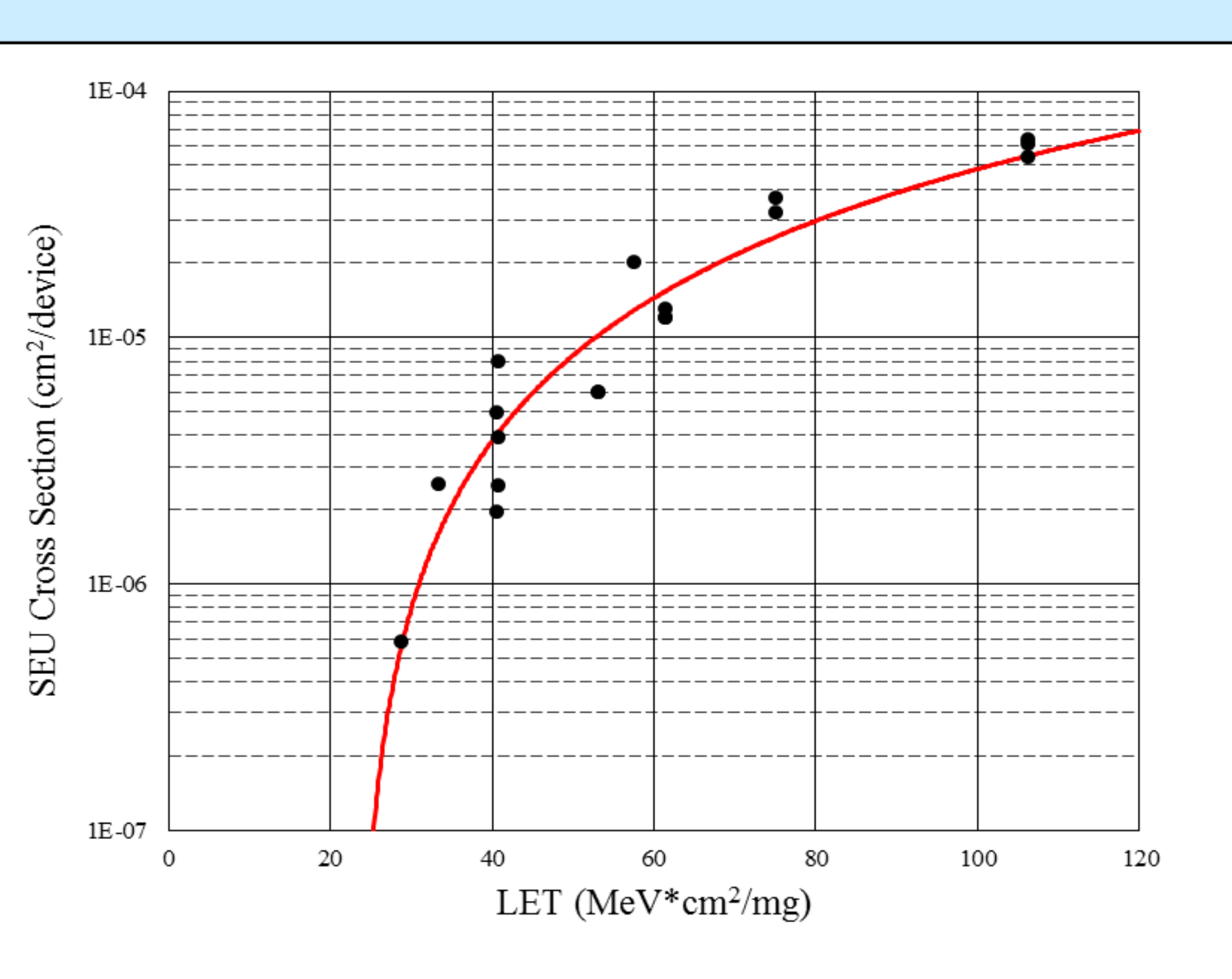

Figure 6: Single event cross section with Weibul fit environments demands thorough understanding of the exvirnmental conditions faced and the device's application-specific and show a susceptibility to

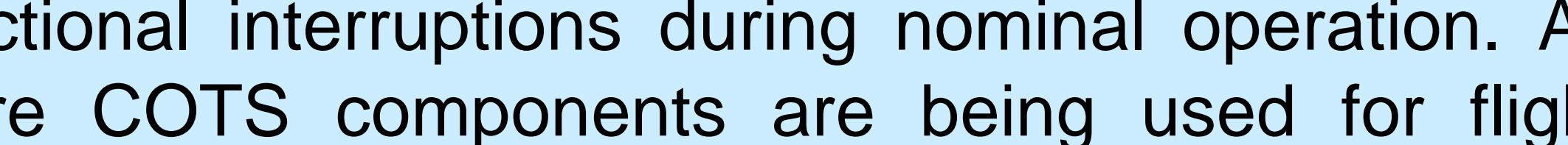
components are being used for tho challenging. The response of the parts depend on the corresponding circuitry when investigating the system In many

e part, or are limited in not trying to characterize hem to do so. By stripping down the intended application to the bare minimum to mimic the can help to understand the radiation For single event effects testing this is in this ele by only bombarding one device at a time. In this experiment we were looking at board leve
response while one device was tested.

References

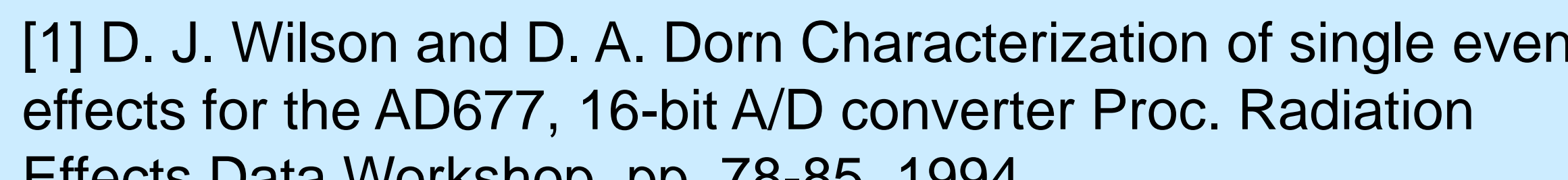
he AD674B", JPL RADATA - [2] "Single Event Effects D
Log NOV1692.SEE, 1992. - [3] D. F. Hoeschele Analog-to-Digital and Digital-to-Analog
Conversion Techniques vol. 1.1994, Wiley-Interscience - 4 I] Sexton, F.W.; Hash, G.L.; Connors, M.P.; Murray, J.R.;
Schwank, J.R.; Winokur, P.S.;; Bradley, E.G., "SEU and SEL

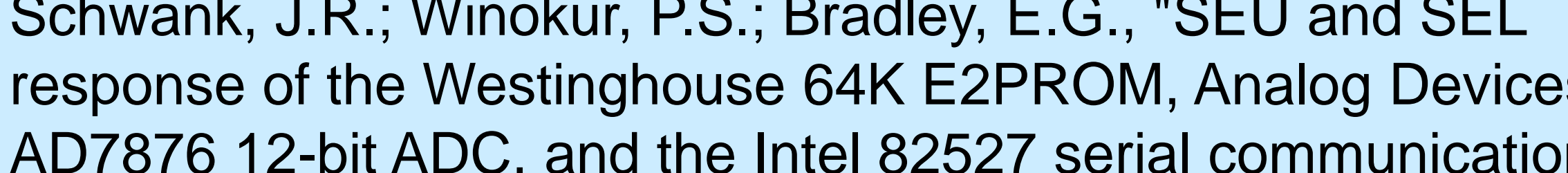
ADD776 12-bit ADC, and the Intel 82527 serial communication
Controller," Radiation Effects Data Workshop, 1994 IEEE, vol., controller," Radiation Effect
no., pp.55,63, 20 July 1994 - [5] hitp:///mww.analog.com/staticlimported-
files/data sheets/AD7984.pdf

Acknowledgments The Authors acknowledge the sponsors of this
effort: Transiting Exoplanet Survey Satellite (TESS) NASA. The authors thank members of the Radiation Effects and Analysis Group (REAG) who contributed to the test results presented here through shipping, test planning, and data reduction: Hak S. Kim
Anthony M. Dung-Phan, Stephen R. Cox, and
Con R 